Article

\title{
Design of a Mechanism with Embedded Suspension to Reconfigure the Agri_q Locomotion Layout ${ }^{\dagger}$
}

\author{
Carmen Visconte, Paride Cavallone $\mathbb{( D}$, Luca Carbonari, Andrea Botta $\mathbb{(}$ ) and Giuseppe Quaglia *(D)
}

check for
updates

Citation: Visconte, C.; Cavallone, P.; Carbonari, L.; Botta, A.; Quaglia, G. Design of a Mechanism with

Embedded Suspension to Reconfigure the Agri_q Locomotion Layout. Robotics 2021, 10, 15. https: / / doi.org/10.3390/ robotics10010015

Received: 10 December 2020 Accepted: 11 January 2021 Published: 13 January 2021

Publisher's Note: MDPI stays neutral with regard to jurisdictional clai$\mathrm{ms}$ in published maps and institutional affiliations.

Copyright: $\odot 2021$ by the authors. Licensee MDPI, Basel, Switzerland. This article is an open access article distributed under the terms and conditions of the Creative Commons Attribution (CC BY) license (https:// creativecommons.org/licenses/by/ $4.0 /)$.

\author{
Department of Mechanical and Aerospace Engineering, Politecnico di Torino, 10129 Torino, Italy; \\ carmen.visconte@polito.it (C.V.); paride.cavallone@polito.it (P.C.); luca.carbonari@polito.it (L.C.); \\ andrea.botta@polito.it (A.B.) \\ * Correspondence: giuseppe.quaglia@polito.it \\ + This paper is an extended version of our paper published in Visconte, C.; Cavallone, P.; Carbonari, L.; Botta, A.; \\ Quaglia, G. Mechanism for the Locomotion Layout Reconfiguration of the Agri_q Mobile Robot. In \\ Proceedings of the 29th International Conference on Robotics in Alpe-Adria Danube Region (RAAD 2020), \\ Kaiserslautern, Germany, 17-19 June 2020; pp. 390-399.
}

\begin{abstract}
The Agri_q is an electric unmanned ground vehicle specifically designed for precision agriculture applications. Since it is expected to traverse on unstructured terrain, especially uneven terrain, or to climb obstacles or slopes, an eight-wheeled locomotion layout, with each pair of wheels supported by a bogie, has been chosen. The wide contact surface between the vehicle and the ground ensures a convenient weight distribution; furthermore, the bogie acts like a filter with respect to ground irregularities, reducing the transmissibility of the oscillations. Nevertheless, this locomotion layout entails a substantial lateral slithering along curved trajectories, which results in an increase of the needed driving torque. Therefore, reducing the number of ground contact points to compare the torque adsorption in different configurations, namely four, six, or eight wheels, could be of interest. This paper presents a reconfiguration mechanism able to modify the Agri_q locomotion layout by lifting one of the two wheels carried by the bogie and to activate, at the same time, a suspension device. The kinematic synthesis of the mechanism and the dynamic characteristics of the Agri_q suspended front module are presented.
\end{abstract}

Keywords: precision agriculture; Agri_q; mobile robot; UGV; agricultural robot; service robotics; sustainability; suspension

\section{Introduction}

Precision agriculture is the management of crop inputs, like water, fertilizer, and plant protection products, at the correct time and place to increase productivity and maximize yields, in a sustainable manner. It requires the cooperation between unmanned aerial vehicles (UAV), which monitor the crops, and unmanned ground vehicles (UGV), which perform the intervention function. Focusing on the UGV, the adaption of commercial machinery as mobile platform for autonomous agricultural vehicles is the most common choice [1-4]. Nevertheless, recently custom mobile robots, which integrate some navigation strategy, have been studied and proposed as universal intelligent platform for various engineering tasks on rough ground [5-10]. Since the capability to move over an irregular and loose soil is one of the mandatory requirements, tracked and the multi-wheeled locomotion layouts are preferred. Tracked mobile robots ensure a wide contact surface between the vehicle and the ground, correctly distributing on the soil the static and dynamic forces acting on the vehicle. On the other hand, complexity and a high power-to-weight ratio characterize this kind of vehicles. Multi-wheeled robots tend to reproduce the ground pressure distribution of track-based systems, with a higher efficiency and a simpler mechanical structure than track-based rovers. These intelligent moving platforms are expected to carry sensitive equipment, like sensors and end-effectors for different tasks, on uneven 
terrain. Therefore, the riding comfort and stability is fundamental and, independently on the chosen locomotion strategy, appropriate suspension systems have to be provided. Some mobile robots adopted the active vibration isolation approach, altering their structures and configurations to change the position of their center of mass when traversing complicated unstructured terrains [11-14]. Nevertheless, expensive manufacturing costs and energy consumption limited the development and application, and passive suspension devices are preferred. A passive bio-inspired limb-like suspension implemented on a truck-based robot is reported in [15]. Some authors report the use of independent suspensions, made up of adjustable gas spring and oil dampened shock absorber, on wheeled vehicles $[16,17]$. Passive articulated suspensions, which permit some of the wheels to rise, relative to the chassis, over bumps without changing the weight distribution or changing it as little as possible, are particularly interesting. A notable example is the so-called rocker-bogie suspension [18] that has been successfully demonstrated by NASA's rovers Spirit and Opportunity for planetary exploration (Maimone et al., 2004) [19].

In [20] the authors report the concept of a novel multi-wheeled electric UGV, named Agri_q, equipped by passive articulated suspensions; each pair of its eight wheels, in fact, is supported by a bogie, which acts like a filter with respect to ground irregularities, reducing the transmissibility of the oscillations. Figure 1 shows the first prototype, characterized by a reconfigurable chassis. The rover, in fact, integrates a positioning mechanism able to compensate longitudinal slopes [21]; this mechanism, together with a motorized roll joint compensating transversal slopes, ensures a horizontal landing surface for drones even when the rover is moving on steep slopes, as shown in Figure 1a. Solar panels cover the landing platform for partial auto recharging of the batteries collection during the passive phases; the previously mentioned positioning mechanism permits to orient the chassis in the most convenient way for solar energy collection. A collaborative robotic arm, exploiting the positioning mechanism to expand its workspace, has been integrated into the rover, to collect ground or leaves samples for lab analysis, as shown in Figure 1b [22].

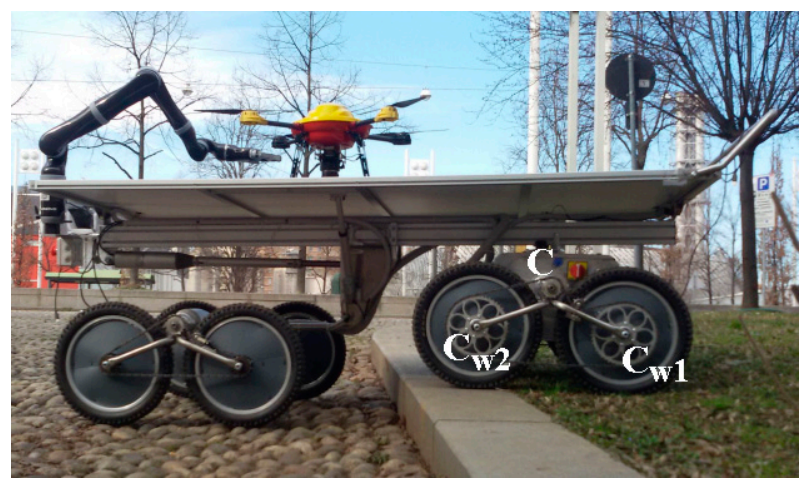

(a)

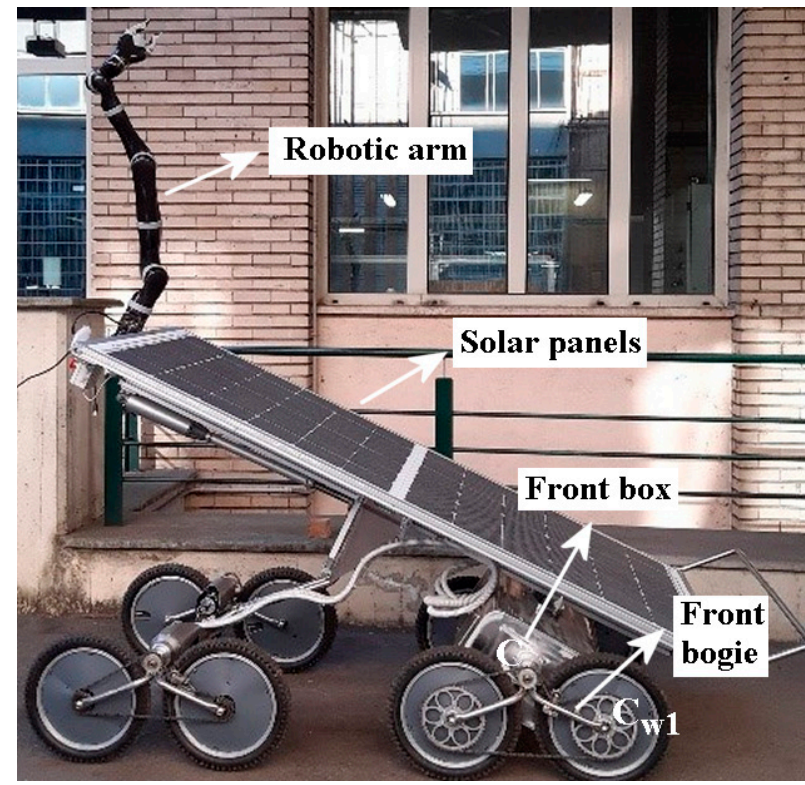

(b)

Figure 1. The Agri_q prototype in its standard 8-wheeled configuration: (a) compensation of longitudinal slopes; (b) expansion of the robotic arm workspace.

Preliminary experimental field tests highlighted that, when the rover engages curved trajectories, a substantial lateral slithering, combined to an increase of the needed driving torque, occurs. Therefore, comparing the torque adsorption in the standard eight-wheeled configuration and in a modified six-wheeled configuration (two front wheels, four back 
wheels, as in Figure 2) could be of interest. In [23] the authors presented a preliminary study of a mechanism able to lift one of the two wheels carried by the rover front bogie. Two possible functional solutions were compared and a kinematic design methodology was presented. Since, in the two front-wheeled configuration, the bogie loses its filter function, the reduction of the vibration transmitted to the sub-chassis was transferred to a commercial suspension system embedded in the reconfiguration mechanism itself, whose dynamics was not yet studied.

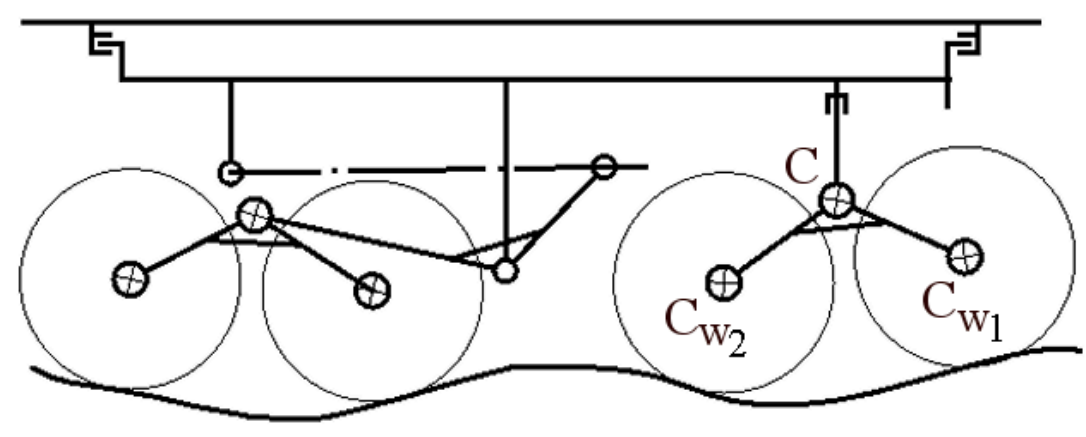

a)

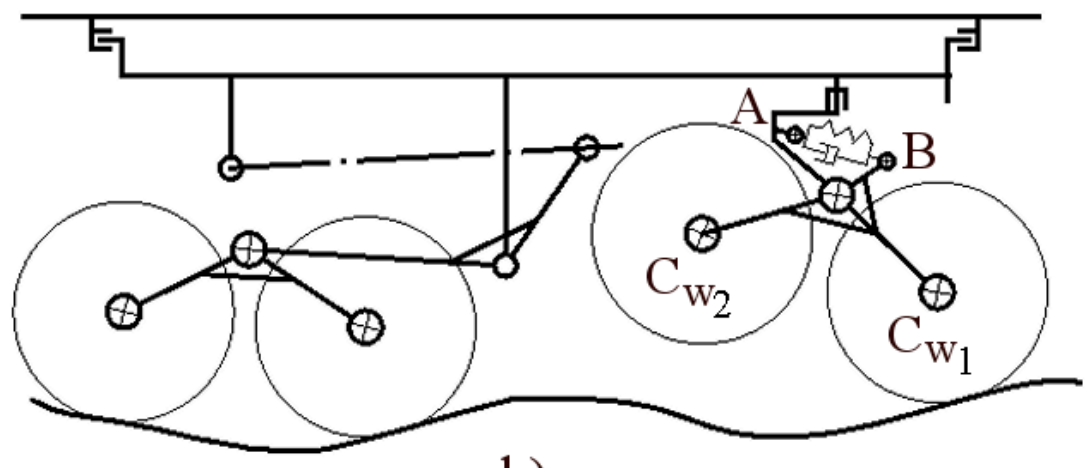

b)

Figure 2. Simplified side view of the Agri_q mobile robot: (a) standard eight-wheeled configuration; (b) six-wheeled configuration, front wheels suspended.

This work presents the locomotion layout reconfiguration system in its final version and focuses on the dynamic behavior of the rover in the two front-wheeled configuration. Firstly, a 2DOF model of the rover suspended front box and a linearized analytical model of the system are presented. Then, results coming from the latter model are compared to those obtained with a numerical approach.

\section{The Reconfiguration Mechanism}

\subsection{Working Principle}

A functional sketch of the proposed reconfiguration mechanism is shown in Figure 3. A four bar-linkage $\mathrm{DCAB}$, driven by a linear actuator linked to the first rocker arm DA, controls the position of the UGV bogie $\mathrm{CC}_{\mathrm{w} 1} \mathrm{C}_{\mathrm{w} 2}$ through its second rocker arm $\mathrm{CB}$. The latter, in fact, is integral with the bogie itself and is pivoted about the joint $C$ fixed on the vehicle front box. In the standard four front-wheeled configuration, the actuator reversibility ensures free rotation of the bogie about the pivot joint $C$; therefore, in the case of a vertical displacement of a single wheel, caused by the contact with an obstacle, a reduced vertical displacement of the whole vehicle is obtained. 


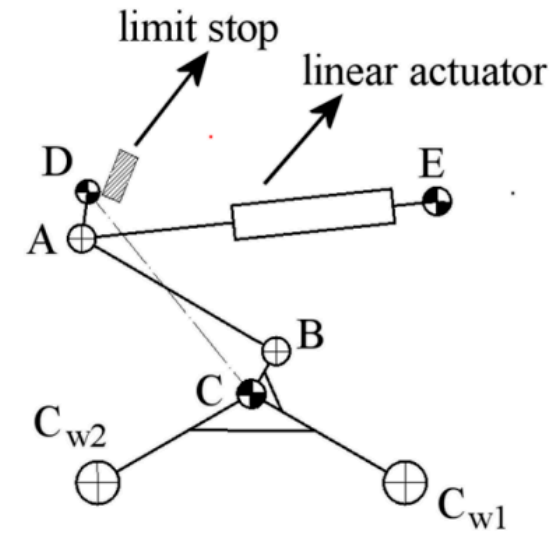

a)

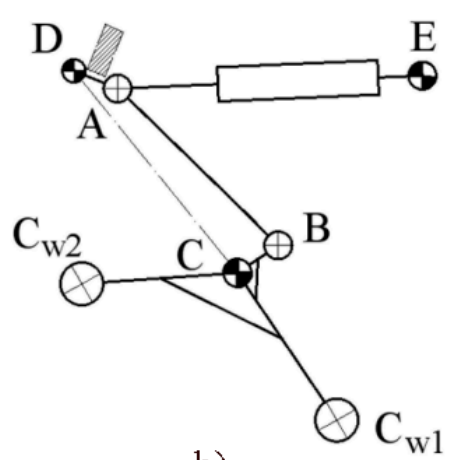

b)

Figure 3. Working principle of the reconfiguration mechanism: (a) four front-wheeled configuration; (b) two front-wheeled configurations.

Enabling the actuator, the link DA goes beyond a dead point and comes finally in contact with a limit stop fixed on the front box; consequently, the bogie rotates about $\mathrm{C}$ and a two-front wheeled configuration is obtained. Since the limit stop prevents further counterclockwise rotations of DA and the force transmitted by the ground ensures a stable configuration of the four-bar linkage, the actuator power supply can be interrupted. Nevertheless, in case of motion over very irregular soil, the link DA could lose its contact with the limit stop; therefore, a brake has to be provided.

In the two front-wheeled configuration, the mechanical filtering of ground irregularities performed by the bogie is lost. Therefore, the coupler link $\mathrm{AB}$ of the proposed reconfiguration mechanism embeds a deformable body. Figure 2 highlights that, when the vehicle is in the two-front wheeled configuration, the hinge A of the reconfiguration mechanism acts like a pivot joint fixed on the front box and the coupler link $A B$ works as a suspension. A rear shock absorber for mountain bike (size $210 \mathrm{~mm}$, spring stiffness $k=131,000 \mathrm{~N} / \mathrm{m}$, damping coefficient $c=1.7 \times 10^{4} \mathrm{~N} \mathrm{~s} / \mathrm{m}$ ) has been chosen as deformable body.

\subsection{Kinematic Requirements}

Figure 4 shows some relevant locations taken by the bogie when the rover locomotion layout is changed; the variable angle $\alpha$ is measured from the vertical direction. In the nominal configuration $\mathrm{CC}_{\mathrm{W} 1 \_} \mathrm{i}$, corresponding to the four front-wheeled layout, the bogie can freely rotate within a range $\Delta \alpha_{i}=26^{\circ}$, to ensure a wide contact surface between the vehicle and the ground even on an irregular terrain.

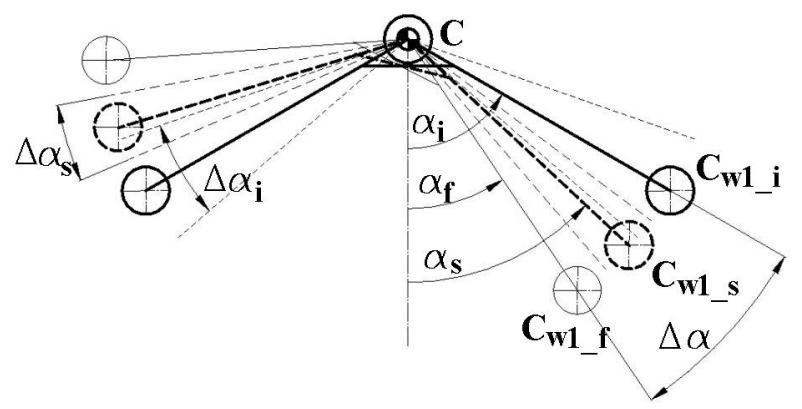

Figure 4. Bogie workspace. Thick continuous line: nominal four front-wheeled configuration; thin continuous line: the suspension device is inserted; thick dashed line: two front-wheeled configuration with the suspension preloaded and ready to work. 
In order to move to a two front-wheeled layout, the reconfiguration mechanism has to place the unloaded bogie arm at the location $\mathrm{CC}_{\mathrm{W} 1 \_\mathrm{f}}$, imposing a rotation $\Delta \alpha$. Finally, because of the effect of the force transmitted by the ground on the wheel, an additional counterclockwise rotation from $\alpha_{f}$ to $\alpha_{s}$ is obtained, which determines the shock absorber preloading. Therefore, at the location $\mathrm{CC}_{\mathrm{W} 1 \_s}$ the suspension device is active and ready to work, within the range $\Delta \alpha_{\mathrm{s}}=26^{\circ}$, which permits a wheel hub vertical displacement of $50 \mathrm{~mm}$.

Figure 5 shows the bogie $\mathrm{CC}_{\mathrm{w} 1} \mathrm{C}_{\mathrm{w} 2}$ in the two front-wheeled static configuration, which corresponds to the equilibrium point between the force $F_{G}$ transmitted from the ground to the wheel, the lifted wheel weight $F_{w}$ and the suspension preload $F_{S 0}$. Considering $h=100 \mathrm{~mm}$ as a reasonable distance between the soil and the lifted wheel and taking into account the shock absorber stiffness, a rotating angle $\Delta \alpha=26^{\circ}$ has to be imposed by the reconfiguration mechanism.

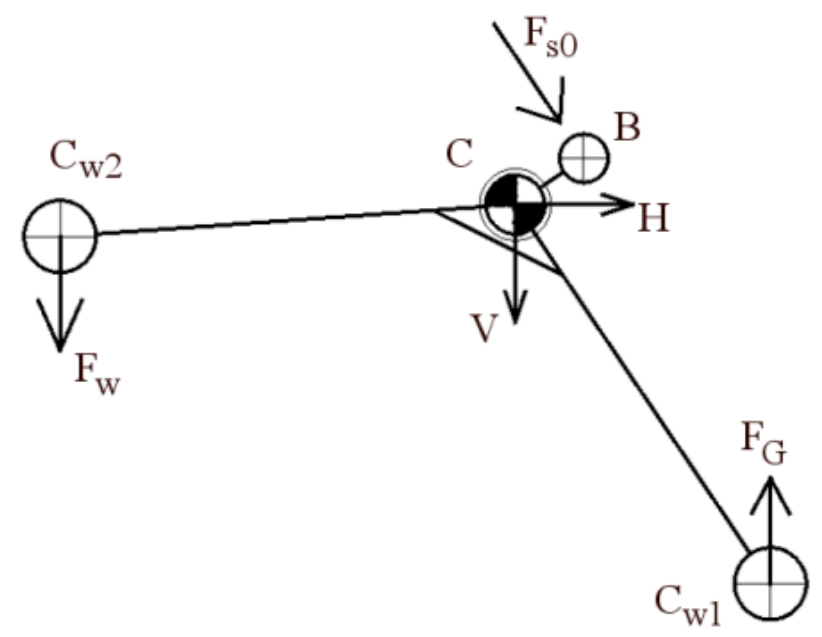

Figure 5. Balance of forces on the bogie $\mathrm{CC}_{\mathrm{w} 1} \mathrm{C}_{\mathrm{w} 2}$.

In the following, the relationship between the desired rotation $\Delta \alpha$ and the needed stroke of the linear actuator is obtained. The kinematic study was carried out considering the reconfiguration mechanism as made up of rigid bodies. In fact, only when the rocker DA reaches the limit stop, the deformability of the coupler link can be exploited.

\section{Kinematic Analysis}

Figure 6 introduces some notations useful for the kinematic analysis. Angles $\delta$ and $\varphi$ are constant parameters that must be properly chosen; they identify the orientation $\delta$ of the frame DC of the reconfiguration mechanism DCAB and the shift angle $\varphi$ between the bogie arm $C_{\mathrm{w} 1}$ and the rocker arm $\mathrm{CB}$, respectively. Angles $\alpha$ and $\gamma$ are variable and define the angular positions of the bogie and of the rocker arm DA with respect to the vertical direction, respectively; $\alpha_{F B}$ and $\gamma_{F B}$ define the same locations, but measured from the reference line DC, so that $\alpha=\alpha_{F B}+\delta-\varphi$ and $\gamma=\gamma_{F B}+\delta$.

\subsection{Amplification of the Output Range of Rotation}

Moving from the four front-wheeled configuration to the two front-wheeled ones, the rocker DA of the reconfiguration mechanism has to overcome a dead point. Therefore, in order to obtain a reliable operation of the mechanism itself and to avoid undesired switches, the two limit positions of the rocker DA have to be kept far to each other. Choosing DA shorter than $\mathrm{CB}$, the rotation $\Delta \alpha$ of the bogie needed to move from one configuration to the other results into a wider rotation $\Delta \gamma$ of the rocker DA, reaching the goal. Furthermore, varying the shift angle $\phi$ between the rocker $C B$ and the bogie arm $C_{\mathrm{w} 1}, \Delta \alpha$ and $\Delta \gamma$ can be placed at a convenient location. Figure 7 introduces the notations needed to determine the analytical relationship between $\alpha_{F B}$ and $\gamma_{F B}$. 


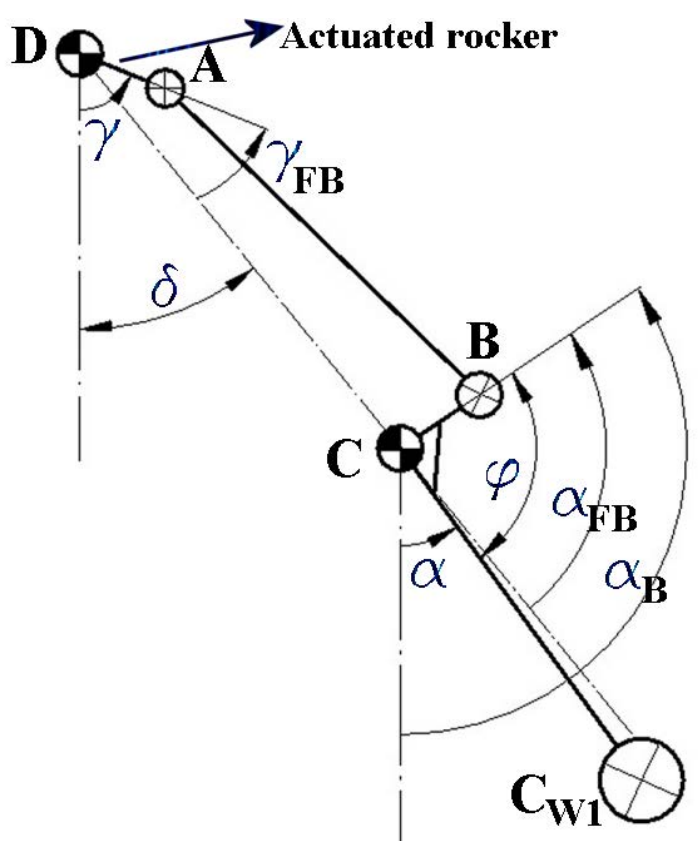

Figure 6. Notations needed to calculate $\gamma=\gamma(\alpha)$.
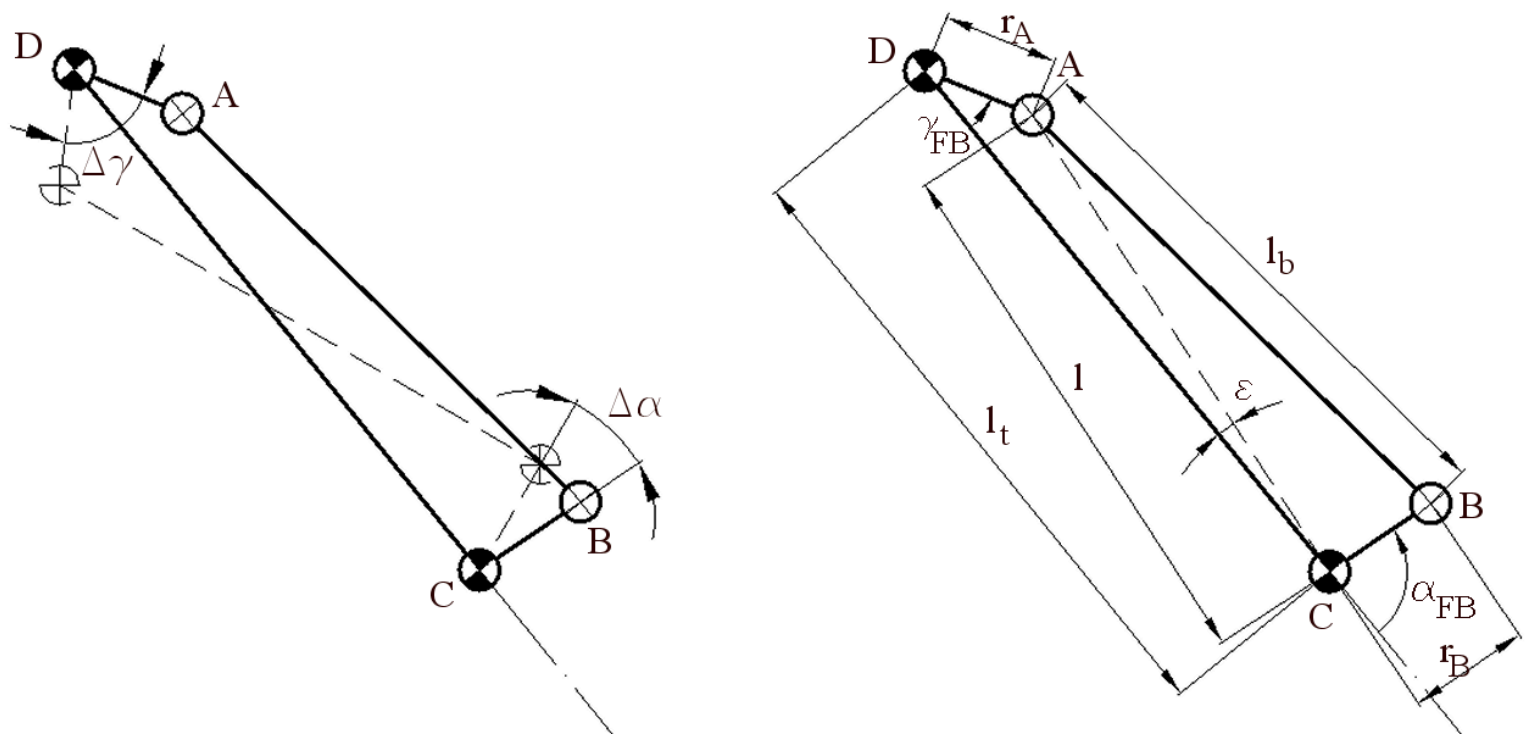

Figure 7. Notations needed to calculate $\gamma=\gamma(\alpha)$.

It can be obtained that:

$$
\alpha_{F B}=\pi-\epsilon-\operatorname{arcos} \frac{l^{2}+r_{B}^{2}-l_{b}^{2}}{2 \cdot l \cdot r_{B}}=\alpha_{F B}\left(\gamma_{F B}\right)
$$

\subsection{Actuation of the Mechanism}

The rotation $\Delta \gamma$ of the link DA can be driven by a linear actuator paired at one end to the front box, by means of the revolute joint $\mathrm{E}$, and paired at the other end to the link DA, by means of the revolute joint F. As shown in Figure 8, the most convenient position of the second revolute joint $\mathrm{F}$ can be chosen, so to reorient $\Delta \gamma$, i.e., to limit the variability of the actuator transmission angle. The same figure highlights graphically the relationship between the desired $\gamma$ rotation and the distance $s_{F}$ between $\mathrm{F}$ and $\mathrm{E}$, i.e., the parameter controlled by the actuator. The angles $\gamma_{A}$ and $\gamma_{F}$ identify the angular position of DA and 
DF with respect to the reference line DE, respectively: $\gamma_{A}=\gamma_{F}+\eta$. The angle $\psi$ is the angular shift of DE with respect to the vertical direction. It is also: $\gamma_{A}=\pi-\psi+\gamma$.
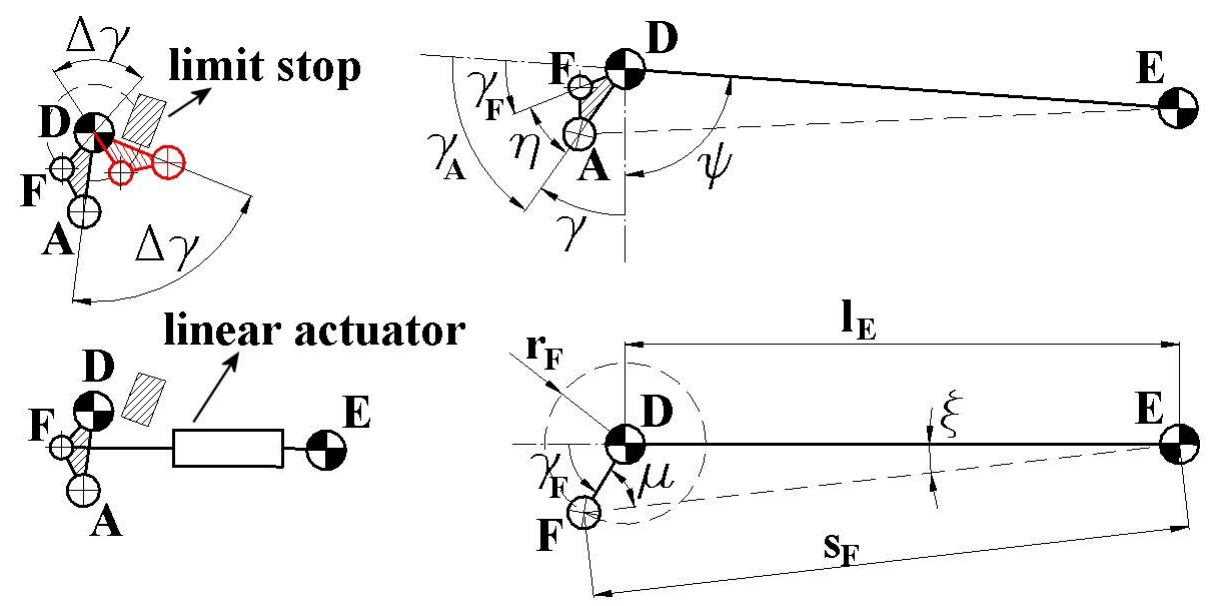

Figure 8. Actuation of the link DC.

According to Figure 8, one has:

$$
\begin{gathered}
l_{E}+r_{F} \cos \left(\gamma_{F}\right)=s_{F} \cdot \cos (\xi) \\
r_{F} \cdot \sin \left(\gamma_{F}\right)=s_{F} \cdot \sin (\xi)
\end{gathered}
$$

For a certain value of the non-dimensional parameter $p_{l}=l_{E} / r_{F}$, using a numerical approach, the angle $\xi$ can be worked out as a function of the input angle $\gamma_{F}$ as follows:

$$
\xi=\arctan \left(\frac{\sin \left(\gamma_{F}\right)}{p_{l}+\cos \left(\gamma_{F}\right)}\right)=\xi\left(p_{l}, \gamma_{F}\right)
$$

In addition, the transmission angle $\mu$ can be worked out as:

$$
\mu=\gamma_{F}-\xi, \text { if }\left(\gamma_{F}-\xi\right) \leq \pi / 2 ; \mu=\pi-\left(\gamma_{F}-\xi\right) \text {, if }\left(\gamma_{F}-\xi\right)>\pi / 2
$$

Introducing the non-dimensional parameter $a_{F}=s_{F} / r_{F}$, it finally follows that:

$$
a_{F}=\frac{s_{F}}{r_{F}}=\frac{\left(p_{l}+\cos \left(\gamma_{F}\right)\right)}{\cos \left(\xi\left(p_{l}, \gamma_{F}\right)\right)}=w_{C}\left(p_{l}, \gamma_{F}\right)
$$

\subsection{Kinematic Results}

Figure 9 shows the results obtained in terms of angular position $\alpha$, instantaneous transmission ratio $d \alpha / d a_{F}$ and transmission angle $\mu$, as a function of the non-dimensional input $a_{F}$. Results are obtained setting DA $=44 \mathrm{~mm}, \mathrm{CB}=46 \mathrm{~mm}, \mathrm{DC}=230 \mathrm{~mm}, \delta=39^{\circ}$, $\psi=87^{\circ}, \mathrm{DE}=400 \mathrm{~mm}, \eta=33^{\circ}, \mathrm{DF}=27 \mathrm{~mm}$, and $\varphi=90^{\circ}$. The latter value of the shift angle was identified by the parametric analysis carried out in [11] as the better compromise between the need to avoid undesired switches of the reconfiguration mechanism and the need of an almost constant instantaneous transmission ratio, useful for motor optimization. 

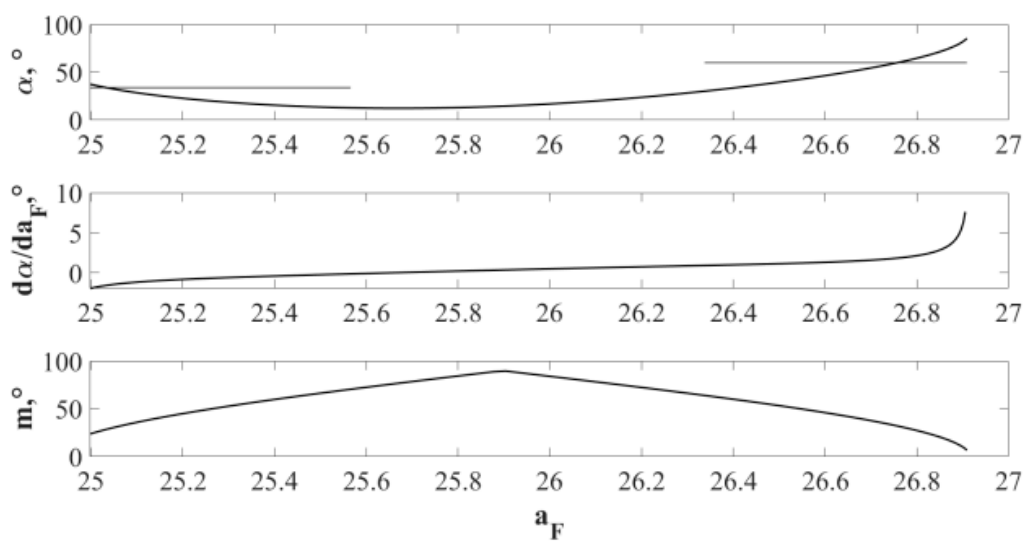

Figure 9. Angular position $\alpha$, instantaneous transmission ratio $d \alpha / d a_{F}$ and transmission angle $\mu$, as a function of the non-dimensional input $a_{F}$.

\section{Dynamic Analysis}

The following analysis refers to the rover in the two front-wheeled configuration: the reconfiguration mechanism is kept in contact with the limit stop, its hinge A acts now like a pivot joint fixed on the front box, and the shock absorber, characterized by a spring stiffness $k$ and a damping coefficient $c$, is preloaded. Taking into account that the front and rear modules of the Agri_q rover are completely independent in terms of roll motion [20,21], the dynamic characteristics of the front suspended module are studied below.

\subsection{Suspension Force Reduction}

In order to study the dynamics of the suspended mass, the force exerted by the suspension device on the swing arm has to be converted into an equivalent force, acting vertically on the wheel hub, as shown in Figure 10. In the figure, $F_{S}$ denotes the force exerted by the shock absorber spring, $F_{s_{-} e q}$ represents the same force reduced at the wheel axle, while $d s$ and $d h$ are the shock-absorber travel and the wheel vertical displacement, respectively.

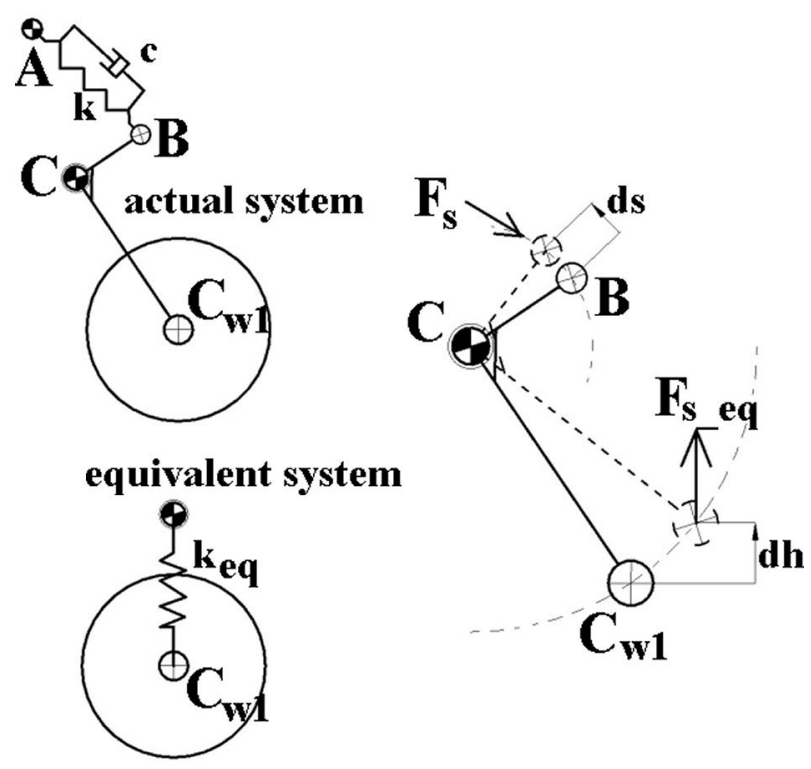

Figure 10. Suspension force reduction at the wheel axle.

Applying the virtual work principle, one has:

$$
F_{s} \cdot d s=F_{s_{-} e q} \cdot d h
$$


Introducing the installation ratio $i_{R}=\frac{d s}{d h}$, which is also the leverage ratio throughout the range of motion, and taking into account that $F_{s}=k \cdot d s$ and $F_{s_{-} e q}=k_{e q} \cdot d h$, it results:

$$
k_{e q}=k \cdot i_{R}^{2}
$$

Similarly, an equivalent damping coefficient can be obtained:

$$
c_{e q}=c \cdot i_{R}^{2}
$$

Figure 11 shows the trend of the suspension equivalent stiffness throughout the range of motion, obtained taking into account the characteristics of the commercial shock absorber and the swing arm geometry. As shown, the suspension device exhibits a nonlinear regressive behavior, acceptable for off-road applications.

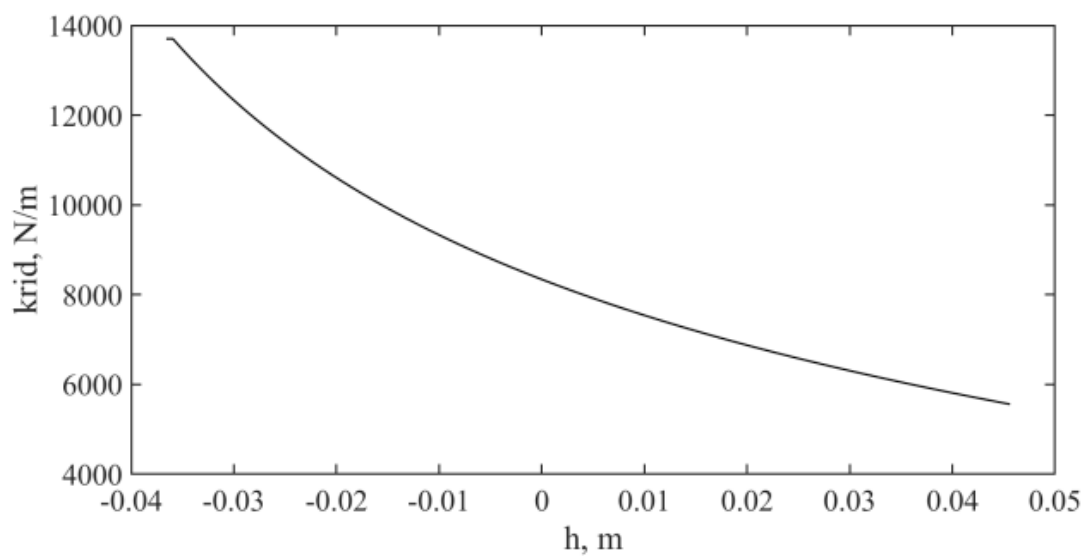

Figure 11. Non-linear characteristic of the suspension equivalent spring.

\subsection{Front Box Model}

The 2-DOF model shown in Figure 12 gives a description of the Agri_q front module roll and bounce dynamics. The motion of the suspended mass, characterized by the mass $\mathrm{m}$ and the mass moment of inertia $I_{x x}$, is described by the vertical position $z_{G}$ and by the body roll angle $\theta_{R}$. The two front suspension stiffness and damping, reduced at the wheel axle, are represented by $k_{e q}$ and $c_{e q}$. The tyre stiffness and unsuspended mass oscillations are neglected. The surface excitation is introduced as the left and right displacements $z_{l}$ and $z_{r}$. The displacements of the points of interest are described in the global coordinate system, whose origin is located on the flat ground.

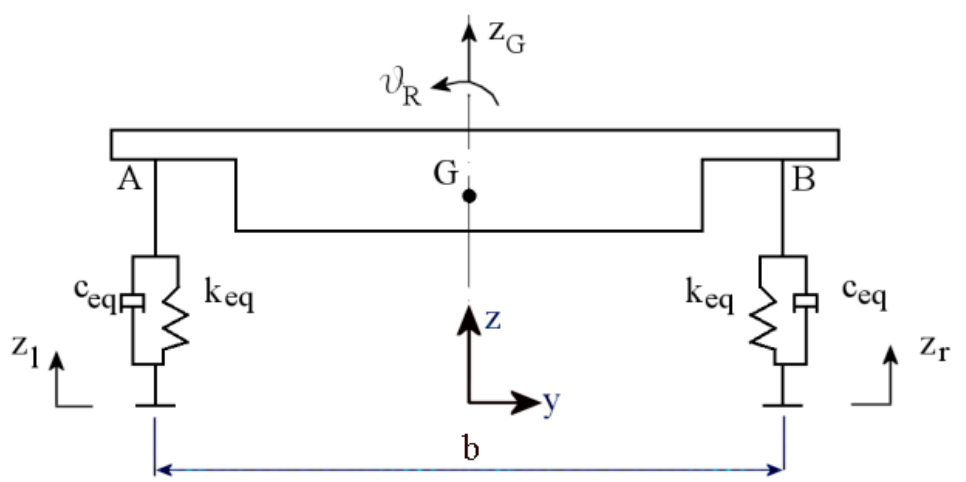

Figure 12. 2-DOF model of the rover front module. 
The locations of the pivot linking the suspended mass to the suspensions depend on the vertical displacement $z_{G}$ and on the roll angle $\theta_{R}$ of the suspended mass itself according to:

$$
\begin{aligned}
& z_{A}=z_{G}-\frac{b}{2} \cdot \vartheta_{R} \\
& z_{B}=z_{G}+\frac{b}{2} \cdot \vartheta_{R}
\end{aligned}
$$

The total kinetic energy of the system is:

$$
T=\frac{1}{2} \cdot m \cdot{\dot{z_{G}}}^{2}+\frac{1}{2} \cdot \dot{\theta}_{R}^{2}
$$

The elastic energy potential is:

$$
V=\frac{1}{2} \cdot k_{e q} \cdot\left(z_{A}-z_{l}\right)^{2}+\frac{1}{2} \cdot k_{e q} \cdot\left(z_{B}-z_{r}\right)^{2}
$$

The Rayleigh function of the energy dissipated in the shock absorbers can be written as follows:

$$
D=\frac{1}{2} \cdot k \cdot c_{e q} \cdot\left(\dot{z_{A}}-\dot{z}_{l}\right)^{2}+\frac{1}{2} \cdot c_{e q} \cdot\left(\dot{z_{B}}-\dot{z_{r}}\right)^{2}
$$

The $i$-th equation of motion has the following Lagrange formulation:

$$
\frac{d}{d t}\left(\frac{\partial L}{\partial \dot{q}_{i}}\right)-\frac{\partial L}{\partial q_{i}}=Q_{i}
$$

where $\{q\}^{T}=\left\{z_{G} \vartheta_{R}\right\}$ are the generalized lagrangian coordinates, $L=T-V$ is the Lagrangian and

$$
Q_{i}=-\frac{\partial D}{\partial \dot{q}_{i}}
$$

In matrix form it is:

$\left[\begin{array}{cc}m & 0 \\ 0 & I_{x x}\end{array}\right]\left\{\begin{array}{c}\ddot{z_{G}} \\ \ddot{\vartheta_{R}}\end{array}\right\}+\left[\begin{array}{cc}2 \cdot c_{e q} & 0 \\ 0 & c_{e q} \cdot \frac{b^{2}}{2}\end{array}\right]\left\{\begin{array}{c}z_{G} \\ \dot{\vartheta_{R}}\end{array}\right\}+\left[\begin{array}{cc}2 \cdot k_{e q} & 0 \\ 0 & k_{e q} \cdot \frac{b^{2}}{2}\end{array}\right]\left\{\begin{array}{c}z_{G} \\ \vartheta_{R}\end{array}\right\}=\left\{\begin{array}{c}c_{e q} \cdot \dot{z}_{l}+c_{e q} \cdot \dot{z}_{r}+k_{e q} \cdot z_{l}+k_{e q} \cdot z_{r} \\ c_{e q} \cdot \frac{b}{2} \cdot \dot{z_{r}}-c_{e q} \cdot \frac{b}{2} \cdot \dot{z}_{l}+k_{e q} \cdot \frac{b}{2} \cdot z_{r}-k_{e q} \cdot \frac{b}{2} \cdot z_{r l}\end{array}\right\}$

As can be seen, the hypothesis of a center of mass located at the middle of the wheelbase results in an uncoupled system of differential equations.

\subsection{Linear Model}

In order to evaluate the system response to an external surface excitation, a linear model of the system has been developed. In particular, the suspension spring characteristic has been linearized around the static preload condition ( $h=0$, in Figure 11), assuming constant values for $k_{e q}$ and, similarly, for $c_{e q}$. Therefore, according to Equation (17), the undamped eigenfrequencies $\omega_{n, z}$ and $\omega_{n, \theta}$ of the bounce and the roll motion, respectively, can be identified as:

$$
\omega_{n, z}=\sqrt{\frac{2 \cdot k_{e q}}{m}} \text { and } \omega_{n, \vartheta}=\sqrt{\frac{k_{e q} \cdot b^{2}}{2 \cdot I_{x x}}}
$$

The corresponding damping factors are:

$$
\zeta_{z}=\frac{c_{e q}}{m \cdot w_{n, z}} \text { and } \zeta_{\vartheta}=\frac{c_{e q} \cdot b^{2}}{4 \cdot I_{x x} \cdot w_{n, \vartheta}}
$$

Referring back to Figure 11, the surface input to the model is described by the displacements $z_{r}$ and $z_{l}$, resulting in an external force $F_{z}$ and moment $M_{\vartheta}$ on the model as:

$$
F_{z}=c_{e q} \cdot \dot{z_{l}}+c_{e q} \cdot \dot{z}_{r}+k_{e q} \cdot z_{l}+k_{e q} \cdot z_{r}
$$




$$
M_{\vartheta}=c_{e q} \cdot \frac{b}{2} \cdot \dot{z}_{r}-c_{e q} \cdot \frac{b}{2} \cdot \dot{z}_{l}+k_{e q} \cdot \frac{b}{2} \cdot z_{r}-k_{e q} \cdot \frac{b}{2} \cdot z_{r l}
$$

Assuming an asymmetric harmonic surface excitation $z_{r}=z_{r 0} e^{i \cdot(w \cdot t)}$ and $z_{l}=0, F_{z}$ can be expressed as:

$$
F_{z}=F_{z 0} \cdot e^{i \cdot(w \cdot t+\chi)}
$$

where:

$$
F_{z 0}=z_{r 0} \cdot \sqrt{\left(\frac{k_{e q}}{m}\right)^{2}+\left(\frac{c_{e q}}{m} \cdot \omega\right)^{2}}
$$

and:

$$
\chi=\operatorname{atan}\left(\frac{c_{e q} \cdot \omega}{k_{e q}}\right)
$$

Similarly, $M_{\vartheta}$ can be expressed as:

$$
M_{\vartheta}=M_{\vartheta 0} \cdot e^{i \cdot(w \cdot t+\chi)}
$$

where:

$$
M_{\vartheta 0}=z_{r 0} \cdot \sqrt{\left(\frac{k_{e q} \cdot l}{2 \cdot I_{x x}}\right)^{2}+\left(\frac{c_{e q} \cdot l}{2 \cdot I_{x x}} \cdot \omega\right)^{2}}
$$

$z_{G}=z_{G 0} \cdot e^{i \cdot(w \cdot t)}$ and $\vartheta_{R}=\vartheta_{R 0} \cdot e^{i \cdot(w \cdot t)}$, the bounce transmissibility $\alpha_{z, r}$ (adimensional) and the roll transmissibility $\alpha_{\vartheta, r}$ (dimensional, $1 / \mathrm{m}$ ) can be calculated as:

$$
\begin{aligned}
& \alpha_{z, r}=\left|\frac{z_{G 0}}{z_{r 0}}\right|=\frac{\sqrt{\left(\frac{k_{e q}}{m}\right)^{2}+\left(\frac{c_{e q}}{m} \cdot \omega\right)^{2}} \cdot e^{i \cdot \chi}}{\sqrt{\left(\omega_{n, z^{2}}-\omega^{2}\right)^{2}+\left(2 \cdot \zeta_{z} \cdot \omega \cdot \omega_{n, z}\right)^{2}}} \\
& \alpha_{\vartheta, r}=\left|\frac{\vartheta_{R 0}}{z_{r 0}}\right|=\frac{\sqrt{\left(\frac{k_{e q} \cdot l}{2 \cdot I_{x x}}\right)^{2}+\left(\frac{c_{e q} \cdot l}{2 \cdot I_{x x}} \cdot \omega\right)^{2}} \cdot e^{i \cdot x}}{\sqrt{\left(\omega_{n, z^{2}}-\omega^{2}\right)^{2}+\left(2 \cdot \zeta_{z} \cdot \omega \cdot \omega_{n, z}\right)^{2}}}
\end{aligned}
$$

The bounce and roll transmissibilities have been analyzed setting $m=59 \mathrm{~kg}, I=2.175 \mathrm{~kg} \cdot \mathrm{m}^{2}$, $b=1 \mathrm{~m}$. Figures 13 and 14 compares the results obtained from the linear model with those obtained numerically, solving the non-linear equation of motion in the Simulink environment. Two different values of $z_{r 0}$, equal to $0.02 \mathrm{~m}$ or $0.2 \mathrm{~m}$, have been imposed to simulate a more or less intense surface excitation. It can be noted that, in case of a high amplitude of the surface excitation, the non-linear numerical model picks the regressive behavior of the suspension device. A suspension that reacts softly to higher amplitude of the surface excitation helps reducing the transmissibility.

\subsection{Roll Vibration Reduction}

Having set $\omega_{n, z}$ conveniently, it is clear from the ratio $\frac{\omega_{n, \vartheta}}{\omega_{n, z}}=\sqrt{\frac{m \cdot b^{2}}{4 \cdot I_{x x}}}$ that there is only a small intervention margin to obtain a $\omega_{n, \vartheta}$ lower than $\omega_{n, z}$ acting on $I_{x x}$. Choosing an appropriate distribution of the masses of the instrumentation contained inside the front box, $I_{x x}$ could be increased without modifying the position of the centre of mass. Nevertheless, according to Equation (17), the most effective way to weaken the roll oscillations would be to limit the difference $\left(z_{r}-z_{l}\right)$. Therefore, in case torque absorption tests on Agri_q would prove that a 6-wheeled configuration is better than the current eight-wheeled configuration, an anti-roll bar could be connected to the Agri_q suspensions. It is worth noting that the introduction of such a device would modify the model under study as shown in Figure 15. 


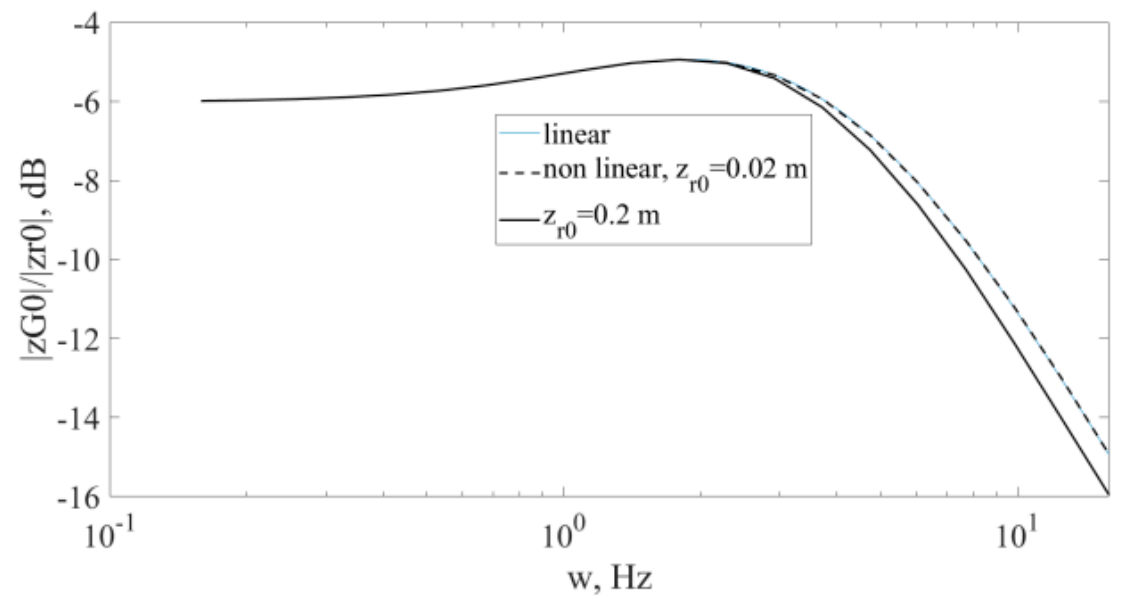

Figure 13. Bounce transmissibility of the Agri_q front box.

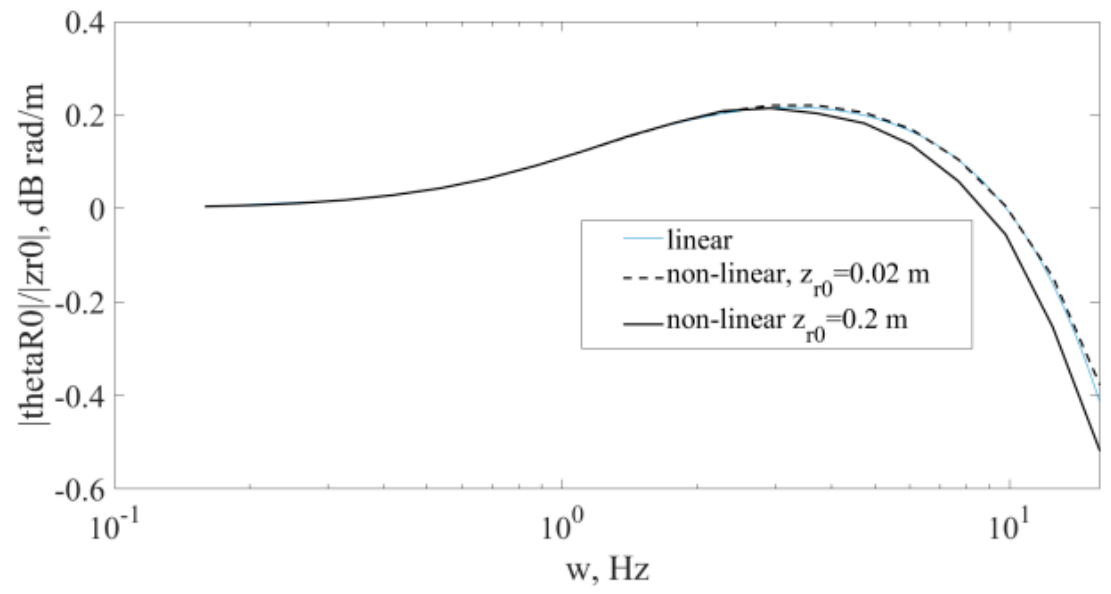

Figure 14. Roll transmissibility of the Agri_q front box.

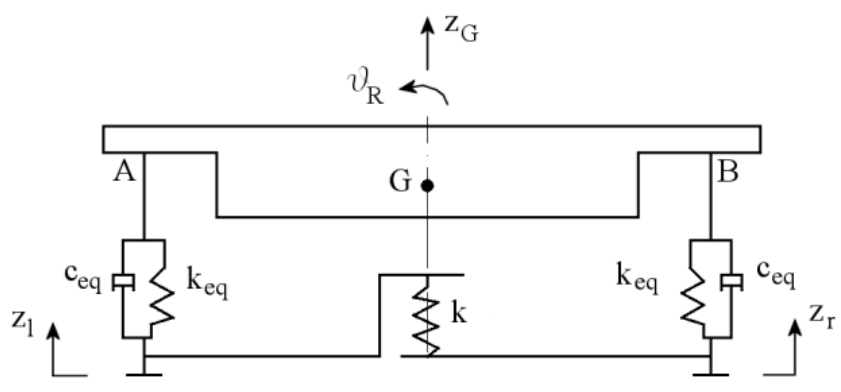

Figure 15. 2-DOF model of the rover front module, with an anti-roll device.

The additional contribution in terms of elastic energy potential is:

$$
V_{\text {add }}=\frac{1}{2} \cdot k \cdot\left(z_{r}-z_{l}\right)^{2}
$$

Therefore, the equation of motion (Equation (17)) and the undamped eigenfrequencies of the system remain unchanged, but the amplitude of the surface excitation for the roll motion is reduced. 


\section{Conclusions}

A mechanism with an embedded suspension device has been studied to reconfigure the locomotion layout of the Agri_q agricultural rover. The mechanism permits to move from a four-front wheeled configuration, in which the ground excitation is filtered by a bogie, to a two front-wheeled configuration, in which a commercial shock absorber reduces the oscillation transmitted to the sub-chassis. Based on the shock absorber characteristic, the mechanism was dimensioned to ensure a reliable operation avoiding undesired switches and providing, at the same time, an almost constant instantaneous transmission ratio, useful for motor optimization. Focusing on the two-wheeled configuration, the device performance in terms of bounce and roll transmissibility to the rover front box has been studied, both by a linear analytical approach and by a non-linear numerical approach. Results suggest providing the rover front box by an anti-roll device, which does not affect the system eigenfrequencies and the system bounce response. In the future, the proposed mechanism will be integrated on the Agri_q prototype and experiments will be carried out to compare different locomotion layouts.

Author Contributions: Conceptualization: G.Q.; Investigation, C.V., P.C., L.C., and A.B.; supervision: G.Q.; writing-original draft: C.V., P.C., L.C., and A.B.; writing-review and editing: G.Q. All authors have read and agreed to the published version of the manuscript.

Funding: This research received no external funding.

Institutional Review Board Statement: Not applicable.

Informed Consent Statement: Not applicable.

Data Availability Statement: The data presented in this study are available on request from the corresponding author.

Acknowledgments: We gratefully thank the PIC4SeR-PoliTO Interdepartmental Centre for Service Robotics.

Conflicts of Interest: The authors declare no conflict of interest.

\section{References}

1. Vidoni, R.; Gallo, R.; Ristorto, G.; Carabin, G.; Mazzetto, F.; Scalera, L.; Gasparetto, A. ByeLab: An agricultural mobile robot prototype for proximal sensing and precision farming. In Proceedings of the ASME 2017 International Mechanical Engineering Congress and Exposition, Tampa, FL, USA, 3-9 November 2017; Volume 4A: Dynamics, Vibration, and Control.

2. Wang, Y.; Lan, Y.; Zheng, Y.; Lee, K.; Cui, S.; Lian, J.-A. A UGV-based laser scanner system for measuring tree geometric characteristics. In Proceedings of the 5th International Symposium on Photoelectronic Detection and Imaging, Beijing, China, 25-27 June 2013.

3. Zaman, S.; Comba, L.; Biglia, A.; Aimonino, D.R.; Barge, P.; Gay, P. Cost-effective visual odometry system for vehicle motion control in agricultural environments. Comput. Electron. Agric. 2019, 162, 82-94. [CrossRef]

4. Shafiekhani, A.; Kadam, S.; Fritschi, F.B.; DeSouza, G.N. Vinobot and vinoculer: Two robotic platforms for high-throughput field phenotyping. Sensors 2017, 17, 214. [CrossRef]

5. Chatzimichali, A.P.; Georgilas, I.P.; Tourassis, V.D. Design of an advanced prototype robot for white asparagus harvesting. In Proceedings of the 2009 IEEE/ASME International Conference on Advanced Intelligent Mechatronics, Singapore, 14-17 July 2009; pp. 887-892.

6. Aljanobi, A.A.; Al-Hamed, S.A.; Al-Suhaibani, S.A. A setup of mobile robotic unit for fruit harvesting. In Proceedings of the 19th International Workshop on Robotics in Alpe-Adria-Danube Region (RAAD 2010), Budapest, Hungary, 24-26 June 2010 ; IEEE: New York, NY, USA, 2010.

7. Tabile, R.A.; Godoy, E.P.; Pereira, R.R.D.; Tangerino, G.T.; Porto AJ, V.; Inamasu, R.Y. Design and development of the architecture of an agricultural mobile robot. Eng. Agrícola 2011, 31, 130-142. [CrossRef]

8. Lin, H.; Dong, S.; Liu, Z.; Yi, C. Study and experiment on a wheat precision seeding robot. J. Robot. 2015, $2015,696301$.

9. Utstumo, T.; Urdal, F.; Brevik, A.; Dørum, J.; Netland, J.; Overskeid, Ø.; Berge, T.W.; Gravdahl, J.T. Robotic in-row weed control in vegetables. Comput. Electron. Agric. 2018, 154, 36-45. [CrossRef]

10. Underwood, J.; Wendel, A.; Schofield, B.; McMurray, L.; Kimber, R. Efficient in-field plant phenomics for row-crops with an autonomous ground vehicle. J. Field Robot. 2017, 34, 1061-1083. [CrossRef]

11. Aoki, T.; Murayama, Y.; Hirose, S. Development of a transformable three-wheeled lunar rover: Tri-star IV. J. Field Robot. 2014, 31, 206-223. [CrossRef] 
12. Michaud, F.; Létourneau, D.; Arsenault, M.; Bergeron, Y.; Cadrin, R.; Gagnon, F.; Legault, M.-A.; Millette, M.; Pare, J.-F.; Tremblay, M.-C.; et al. AZIMUT, a leg-track-wheel robot. In Proceedings of the 2003 IEEE/RSJ International Conference on Intelligent Robots and Systems (IROS 2003), Las Vegas, NV, USA, 27-31 October 2003; pp. 2553-2558.

13. Grand, C.; Benamar, F.; Plumet, F.; Bidaud, P. Stability and traction optimization of a reconfigurable wheel-legged robot. Int. J. Robot. Res. 2004, 23, 1041-1058. [CrossRef]

14. Jiang, H.; Xu, G.; Zeng, W.; Gao, F.; Chong, K. Lateral stability of a mobile robot utilizing an active adjustable suspension. Appl. Sci. 2019, 9, 4410. [CrossRef]

15. Sun, B.; Jing, X. A tracked robot with novel bio-inspired passive "legs". Robot. Biomim. 2017, 4, 18. [CrossRef] [PubMed]

16. Grimstad, L.; From, P.J. The Thorvald II agricultural robotic system. Robotics 2017, 6, 24. [CrossRef]

17. Bawden, O.; Ball, D.; Kulk, J.; Perez, T.; Russell, R. A lightweight, modular robotic vehicle for the sustainable intensification of agriculture. In Proceedings of the Australian Conference on Robotics and Automation (ACRA 2014), Melbourne, Australia, 2-4 December 2014; Australian Robotics \& Automation Association ARAA: Broadway, Australia, 2014.

18. Bickler, D. Roving over Mars. Mech. Eng. 1998, 120, 74-77. [CrossRef]

19. Maimone, M.; Johnson, A.; Willson, R.; Matthies, L. Autonomous navigation results from the Mars exploration rover (MER) mission. Exp. Robot. 2006, IX, 3-13.

20. Quaglia, G.; Cavallone, P.; Visconte, C. Agri_q: Agriculture UGV for monitoring and drone landing. In Mechanism Design for Robotics-Mechanism and Machine Science; Gasparetto, A., Ceccarelli, M., Eds.; Springer Nature Switzerland AG.: Cham, Switzerland, 2019; Volume 66, pp. 413-423.

21. Quaglia, G.; Visconte, C.; Scimmi, L.S.; Melchiorre, M.; Cavallone, P.; Pastorelli, S. Design of the positioning mechanism of an unmanned ground vehicle for precision agriculture. In Advances in Mechanism and Machine Science-Mechanism and Machine Science; Uhl, T., Ed.; Springer Nature Switzerland AG.: Cham, Switzerland, 2019; Volume 73, pp. 3531-3540.

22. Quaglia, G.; Visconte, C.; Scimmi, L.S.; Melchiorre, M.; Cavallone, P.; Pastorelli, S. Robot arm and control architecture integration on a UGV for precision agriculture. In Advances in Mechanism and Machine Science-Mechanism and Machine Science; Uhl, T., Ed.; Springer Nature Switzerland AG.: Cham, Switzerland, 2019; Volume 73, pp. 2339-2348.

23. Visconte, C.; Cavallone, P.; Carbonari, L.; Botta, A.; Quaglia, G. Mechanism for the locomotion layout reconfiguration of the Agri_q mobile robot. In Advances in Service and Industrial Robotics-Mechanism and Machine Science; Zeghloul, S., Laribi, M.A., Arevalo, S.J.S., Eds.; Springer Nature Switzerland AG.: Cham, Switzerland, 2020; Volume 84, pp. 390-399. 